\title{
The Value of Antique Music Score in Li Bai Poem
}

\author{
Yanshuang Hou \\ Libai Research Center \\ The Institute on Libai of Sichuan Province \\ Mianyang, China 621000 \\ School of Music \\ University of Jinan \\ Jinan, China 250022 \\ School of Education \\ Tianjin University \\ Tianjin, China 300072
}

\author{
Xiaona Zhao \\ School of Music \\ Mianyang Normal University \\ Mianyang, China 621000
}

\author{
Xinglong Guo* \\ Libai Research Center \\ The Institute on Libai of Sichuan Province \\ Mianyang, China 621000 \\ *Corresponding Author
}

\begin{abstract}
Researching on $\mathrm{Li}$ Bai poem and music is mainly focused on aesthetics, history and literature. Tang dynasty poem and music are inter-dependent and mutually promoting. Many studies concern on the legendary personality charm of $\mathrm{Li}$ Bai and some other respects, such as cultural characteristics, thought connotation and cultural influence of poetry. However, the historical trace of AMS (Antique Music Score) in Li Bai poem was obviously ignored. People concluded that $\mathrm{Li}$ Bai poem can be sung through analyzing Absolutely Sentence (one kind poetry form, Jue Ju) of Tang Dynasty poetry, the relationship between $\mathrm{Li}$ Bai poem and music as well as sing elements of $\mathrm{Li}$ Bai poem. In the paper, the path discovery of AMS of Li Bai poem was introduced and the simple examples of AMS of Li Bai poem were given, which strongly prove that $\mathrm{Li}$ Bai poem can be sung and there are obvious characteristics of music elements in $\mathrm{Li}$ Bai poem. And because of original relationship of $\mathrm{Li}$ Bai poem and AMS, Li Bai poem can be widely spread and keeping fresh vitality. It proposes some revelations about music feature by music performing form companied song with dance, changeable music rhythm and national characteristic dance styles. Singing Li Bai poem based on AMS provides some new original elements and paths for $\mathbf{L i}$ Bai research, which possess a positive contemporary value.
\end{abstract}

Keywords-musical characteristic; Li Bai poem; relationship of poetry and music; Antique Music Score (AMS)

\section{INTRODUCTION}

\section{A. Evaluation of Li Bai and Poetry}

Li Bai (701-762), nicknamed Tai Bai, was one of the great poets in Tang dynasty acclaimed from his own day to

The Social Science Planning Research Project of Shan Dong province No.15CWYZ33) and the Li Bai Culture Research Center (Key research base on humanities and social science of Sichuan Provincial Department of Education, No.LB2016-001) for funding required to carry out this work the present as a genius and a romantic figure who took traditional poetic forms to new heights, and also titled as a "Poem God". It is recorded that there are about nine hundred ninty poems created by $\mathrm{Li}$ Bai. Li Bai poems conquered many people from government officials to civilians at that time, and he won the high prestige and status. Among the history of poem, Li Bai is one of the most prominent figures in the flourishing of Chinese poetry in the Tang Dynasty.

Li Bai collected the classical poem and opens a new world first. It mainly displays these two aspects: personality charm and poetry artistic achievement. In terms of personality glamour, it shows his massive and youth enthusiasm, positive and optimistic life attitude, strong individual character as well as serving the country's patriotism in his poetry. Some great poets like Su Shi and Lu You, who have been influenced by him. Du Fu once described Li Bai poem like this: the wind and rain were shocked when the pen put down and the finished poetry could make the gods cry". In the regard to the artistic achievement of poem, his works make the later countless poets worship them. Li Bai initiated the romantic style of poetry in Tang Dynasty because of the peculiar and unpredictable imagination, the splendid and beautiful image, the fresh and lively language, the bold and elegant style as well as the strong and romantic art style in his poems. His poetry level has reached the peak in Tang Dynasty. Numerous poems of Li Bai have been successively translated into many different languages spread in other countries, which makes the world realize the charm of Li Bai and his poems. 


\section{B. Translation of Li Bai Poem Expanded Its International Spread}

Chinese and foreign poetry mutual influence and infiltrate in the history of cultural exchange between China and other countries. The impact of tang poem on the rise of American new poetry movement and imagist poetry has been significant. The earliest man on translation of Li Bai poem is the western missionaries, scholar and poet, which gets Li Bai poems spread around the world. Although there are a lot of people to advocate $\mathrm{Du} \mathrm{Fu}$ and deny $\mathrm{Li}$ Bai since the Song Dynasty, it seems to be no affect in the western. Even at the beginning of the translation, in spite of the substantial mistranslations and missing translations, Li Bai poem has been widely spread and loved. No matter the scholar, poet and general readers, they consider him as the delegate of the Chinese poem, and even the Oriental culture. Poetry itself style as fresh and lively language, magnificent and beautiful imagination, as well as romantic and unconstrained, especially Confucianism in his poem also attracts their attention.

The second half of 20th century, there are large numbers of outstanding Chinese scholars and translators in the translation team of Li Bai poem, such as Liu Shi Shun, Luo Yu Zheng, Weng Xian Liang, Xu Yuan Chong, Xu Zhong Jie and Sun Da. Among them, Xu Yuan Chong published an English album - "selected poems of Li Bai" done by the Chinese themselves, who is a translator after "the poet of $\mathrm{Li}$ Bai" of Arthur Waley, "selected poems of Li Bai" of Shigeyoshi Obata as well as "Li Bai and Du Fu" of Arthur cooper [1]. He conducted the precious test and breakthrough in the translation practice of $\mathrm{Li}$ Bai poem from "difficult Shudao", "antique nine poems" and "valiant", and made outstanding contributions to seek the worldwide research of Li Bai poem.

\section{Case in International Translation of Li Bai Poem}

Tang poetry spreads wildly. There have been many foreign langue translations, such as English, French, German, Italian, Japanese language and so on (one representative translation in Table 1). Li Bai and his poetry is introduced to Europe by Jean Joseph Marie Amiot, a Jesuit missionary in Beijing. In the 18th century, translations of his poems began to appear in Europe. The poems were models for celebrating the pleasures of friendship, the depth of nature, and the happiness of drinking. Among the most famous are "The Hard Road to Shu", and "Quiet Night Thought", which still appear in school texts in China today. In the West, multilingual translations of Libai's poems continue to be made. As for the publication and influence of tang poetry around the world, it has been made an extremely high evaluation by the world's encyclopedia. For example, an item about Li Bai said "Li Bai and Du Fu are the world-recognized great poets in China and their poetry give people with boundless beauty", which was written by Ronald of Michigan University cited from the encyclopedia of United States volume 12 version 1975 [2].There are still many short poems inscribed Li Bai in the west except the long ones. Although some discrepancies exist on the content and thought with the original poems of $\mathrm{Li}$ Bai, it reflects the love and acceptance to Li Bai and his poetry in the west.
As early as 1780, an English poet John Scott wrote down a long poem to praise $\mathrm{Li}$ Bai (Li Bai, perhaps a good officer, a song of Chinese pastoral) based on the interpretation of Confucianism in the Li Bai poem. Two centuries later, an American poet Conrad Aiken also depicted a long poetry (A Letter from Li Bai) with the theme of Li Bai. These two articles (The Flourishing Period of Chinese Poetry and the poems of Tang and Song Dynasty) written by Alfred Fokker, translate and introduce 27 poems of Wang Wei, Du Fu and Li Bai. A German scholar Heilman (He praised that the Chinese lytic poetry since 12th century is a sensational translation compiled by Hans Heilman) wrote down the Chinese Ancient and Modern lyric poetry, which translated and introduced 24 poems of $\mathrm{Li}$ Bai. Klabund totally translated 44 poems in Li Bai collection of poems. These books are very prominent to the translation of $\mathrm{Li}$ Bai poems, such as Metaphor of Li Tai Bai Poetry and Poetry of Li Tai Bai translated by E.Von Zach, and Orient backbone: the story of poet Li Bai translated by Kurt Eggers. The Chinese Poem is the first Chinese poetry to be selected and translated into Russian published at 1911 in Russia, including Li Bai, Du Fu, Meng Haoran and Wang Changling. A French scholar Judith Gautier interpreted and published the ancient poetry anthology of China named Le Livre de Jade in 1869 which was later translated into many languages [3]. In 1897, Le Livre de Jade was translated into English by an American poet Stuart Merill.

Research of the relationship between Li Bai poem and music mainly focused on aesthetics, history, literature etc. In China although research of $\mathrm{Li}$ Bai poem occasionally touched in music, but there is not analysis on musical attribute classification and music characteristic of $\mathrm{Li}$ Bai poem and music. In process of international spread and translation of $\mathrm{Li}$ Bai poem, many studies focus on $\mathrm{Li}$ Bai legendary personality glamour, cultural characteristics, thought connotation, cultural influence power of poetry. But it seems that there is not music factor and ignored the historical trace of AMS (Antique Music Score) of Li Bai poem.

The research about the relationship between Li Bai poem and music mainly focused on aesthetics, history and literature. In China, the research on $\mathrm{Li}$ Bai poem occasionally touches music, but there is no analysis on music property classification and music characteristic between $\mathrm{Li}$ Bai poem and music. In the process of translation of $\mathrm{Li} \mathrm{Bai}$ poem and international spread, many studies only pay attention to the legendary personality glamour of $\mathrm{Li}$ Bai, cultural characteristics, thought connotation and cultural influence of poem. But it seems that there is no music element in Li Bai poem, and the historical trace of AMS in $\mathrm{Li}$ Bai poem is ignored.

In conclusion, it reveals that the excavated and reorganized AMS will provide the latest developments for researchers worldwide, through the study on the relationship between $\mathrm{Li}$ Bai poem and music.

\section{Demonstration OF Li Bai Poem Being Sung}

Research of the relationship between Li Bai poem and music mainly focused on aesthetics, history, literature etc. 
Although have studied esthetics thought of Li Bai poem from of aesthetics angle, have studied relationship between Li Bai poem and Ancient Folk Songs from historical perspective, have studied musical attribute of Li Bai poem from literary angle, but lack professional analysis of pure musical elements in musical rhythm, musical melody, composition, performance etc.

\section{A. Absolutely Sentence of Tang Dynasty Poetry Be Sang}

Many researchers consider that Absolutely Sentence (one kind form of poetry, Jue Ju) exactly is musical office of Tang dynasty, it may be sung. No accurate historical record to prove that Absolutely Sentence be sang is Musical office. Wang zhuo (Song dynasty people) consider Tang Dynasty poetry as Bamboo Branch (Zhu Zhi), Wave Rinse Sand(Lang Tao Sha), Happy for Throwing Ball(Pao Qiu Le), Willow Branch (Yang Liu Zhi) is Absolutely Sentence of poetry, and was identified as song. Many poetry of that time also are written according to melody. The poetry can be sung and matched music is called Tang Sound poem (Tang Sheng Shi) by Mr Ren Ban Tang. Qingping Tone Word (QingPing Diao Ci) 3 chapter of Li Bai also is Absolutely Sentence (Jue Ju). Yet most Absolutely Sentence (Jue Ju) of Li Bai especially has characteristics of lyrics. It can't reach such high level if is not affect and nourish of Flourishing Tang dynasty poetry. Song poem musical office (Yue $\mathrm{Fu}$ ) has a natural relationship with music.

Li Bai wrote very fresh and natural in his five words and seven words of Absolutely Sentence (Jue Ju). It is easy to understand. Have Folk songs style but profound and lasting of Charm. There are a lot of songs can be sang in Li Bai Album. For example, Tang Sound poem (Tang Sheng Shi) of Li Bai has Sariputta ( She Li Fei), Ma Duo Lou Zi(title of a poem On the frontier by a Buddhist music piece), Zui GongZi (one of miscellaneous song songs), huan sha shi shang nv, Yu Tian Cai Hua(Yu Tian, today hetian area in Xinjiang). The Midnight Song(ZhiYe Si Shi Ge-Four songs) etc. Songs of the Southern Lass ( Yue Nv Ci)-No.3: A maiden gathers lotus in the creek; Singing, she turns round, seeing passers-by. Smiling, she hides mid lotus blooms her cheek. And won't appear again: she seems so shy. Song of Ba Woman: The River fast like arrow flows, your boat as if on wings swift goes. Ten months like as a thousand miles away, when will you comeback, on what day? And Moon songs over the Mount Emei (E Mei Shan Yue Ge): The crescent moon on the Mount Emei looks like half of moon autumn. Its deep reflection flows into limpid river water. I'll leave the town on Clear Stream for Three Gorges now. I miss you but you are out of view in Yuzhou. Seeing Men hao ran off Yellow Crane Tower to Guanling (Huang He Lou Song Meng Hao Ran Zhi Guang Ling): My friend has left the west where the Yellow Crane Tower, For River Town green with willows and red with flowers. His lessening sail is lost in the boundless azure sky, Where I see but the endless River rolling by. Give to Wang Lun while my farewell(Zeng Wang Lun) : LiBai, sit aboard a ship about to go, When suddenly on shore your farewell songs overflow. However deep the Lake of Peach Blossoms may be, it is not as deep as Wang Lun's friendship. Above wonderful describing is only extracted. Li Bai wrote very fresh and natural in his five words and seven words of Absolutely Sentence (Jue Ju). It is easy to understand. Have Folk songs style but profound and lasting of Charm. There are a lot of songs can be sang in $\mathrm{Li}$ Bai Album.

$\mathrm{Li}$ Bai poem is famous as its ancient poetry and Absolutely Sentence (Jue Ju). Ancient poetry is more freedom in poetry rule, can reflect author say words and dialect. Li Bai love traveling and trip to Shanxi, Shandong, Anhui, Hunan, Henan of province etc. He is willing to contact with local folk artists, and absorbed nutrition of local folk songs. So Li Bai long and short forms of ancient poetry are suitable to writing and singing of folk songs too. His Musical Office poetry and Absolutely Sentence (Jue Ju) are widely spread and singing. There is more than 150 songs Musical Office poetry in his poetry anthology. These musical office (Yue Fu) poems is new poem be written by modeled Ancient musical office (Yue Fu) style, and it can be chant but not be sang. In fact, some musical office poems can be sang, these poems is mostly Absolutely Sentence (Jue Ju). Li Bai Absolutely Sentence (Jue Ju) has characteristic of sincere feeling and rich of folk song style. Li Bai regard poem as song, has open music spirit and bold style, reflects tendency of music of Li Bai poem.

\section{B. Relationship of Li Bai Poem and Music}

Poetry and music can be linked together. This has been proved in cultural heritage of world. For example, Homer's Epic (Ancient Greece) is transmitted by means of singing. Elling O.Eide (American scholar) deeply research Li Bai Complex Word- song poem musical office (Yue Fu )(Za Yan, a type of classical Chinese poetry) of Musical Office poetry. $\mathrm{He}$ considered that changing rhyme and irregular rhythm is related to Central Asian music in Tang dynasty. For relationship between $\mathrm{Li}$ Bai poem and music, focused on itself music attributes, relevance of poetry and folk songs, aesthetic angle of view in Li Bai's poem.

First, in Li Bai poem and Flourishing Tang dynasty music (Ge jing chun, 1995), he pointed out, Some long song poem musical office (Yue $\mathrm{Fu}$ ) of Li Bai is similar with Da qu and Fa qu of Flourishing Tang dynasty China, rich changing in layout structure and rhythm. The Song Rhetoric (Ge Ci) of musical office is originally song word be sang. So it tightly connected with music. Yet song poem musical office (Yue Fu) of Li Bai came from Ancient musical office (Yue Fu) or wrote poem by modeled Ancient musical office (Yue Fu) or protocoled old title with changing. It is easy to see its blood relationship with Ancient musical office (Yue Fu) [4].

Second, Relating Criticism of Li Bai Musical Rhetoric (Li Bai Yue Ci Shu Kao, Ji WenBin, 2008), Discuss Rhythm of Li Bai poem (Xiu Xiang meng, 2010), Inner Characteristics of Music in Li Bai poem (Chang Dekun, 2014), these article dig poems with musical trace be recorded in Tang sound poem (Tang Sheng Shi), Poetry Anthology of Musical office (Yue Fu Shi Ji). And summarized characteristics of local folk music and $\mathrm{Li}$ Bai poem, described oral expression of Li Bai Song Rhetoric, points out condition of select word and matching music, according to melody to fill rhetoric, according to word to fill rhetoric. 
Third, Chinese musical literature history (Zhu Qian Zi, 1935), Tang sound poem up and down (Ren Ban Tang, 2006), Research of Sui-Tang (one name of dynasty) Five Dynasties Wallow (Yan) Music Miscellaneous Words (Za Yan), Song Rhetoric(Ge Ci) (Wang Kunwu, 1996), these papers demonstrated that Tang poetry transmit musical beauty by describing sound, and itself language of Tang poetry contain music. Authors try to describe original features of Chinese ancient poetry and its developing rules.

Fourth, Tang Poetry and Music (Chen Shi Ping, 2001), article discussed relationship between Tang poetry and music from aspects of Tang poetry and lyrics, Tang poetry and Folk song.

Fifth, Thought No Evil-Research of Chinese Literati Music Thought (Chen Si Hai, 2002), Simple Analysis of Musical Aesthetic Thoughts in Whole Tang Poetry (Hou Shuang Xia, 2013), Research of Chinese Poetry Art (Yuan Xing Pei, 2008), Whole Tang Poetry (Zhong ChongBin, 2010) etc. these articles researched musical aesthetics thought of Flourishing Tang dynasty period's poets Li Bai Wang Wei, Du Fu and Bai JuYi from angle of music aesthetics. And particular custom of poetry art, artistic style and artistic achievement also is analyzed in these articles.

\section{Music and Singing Elements in Li Bai Poem}

About music and singing of Li Bai poem, research on four aspects, with musical instruments accompaniment, without musical instruments accompaniment, singing of word poem (Ci Yue), carding existing AMS (Antique Music Score) of Li Bai poem. Relationship between of ancient Chinese music and $\mathrm{Li}$ Bai poem to deduce singing characteristics is studied to paint original features in musical creation, chanting, singing of Li Bai poem [5]. Musical trace of $\mathrm{Li}$ Bai poem is explored by digging musical elements, carding existing AMS (Antique Music Score), analysis musical environment of Li Bai poem [6].

Music in Song poetry of Li Bai Musical Official poetry is given priority to north music. Whole appreciation characteristic of beauty mainly has wide musical range, changeable rhythmical forms, warm and bold and unconstrained [7]. Elementary analysis of itself music was finished in melody style of musical scale, rhythm and beat, prime tone, material of composition, pattern of end etc [8]. Chanting and singing of Chinese classical poetry (Fu Xueyi, 1994), Research of Music and Dance Poetry of Tang Dynasty (Yang ming, 2014), Blow-Playing musical poetry of Tang Dynasty (Lu Yun, 2013), Research of Pipa Poetry of Tang Dynasty (Yu Xiao xue, 2013), musical constituting elements as blow-playing music, music-dance be described in Flourishing Tang dynasty poetry were analyzed in these articles.

To sum up, although many Song Rhetoric of Ancient musical office (Yue Fu) already cannot be sang in Tang Dynasty China, but relationship between poetry and musical dance is very intimate because of Song Rhetoric of Ancient musical office (Yue Fu) even can be sang. Yet Musical office (Yue $\mathrm{Fu}$ ) poem of $\mathrm{Li}$ Bai originate from Ancient musical office (Yue Fu) or wrote poem by modeled Ancient musical office (Yue $\mathrm{Fu}$ ) or protocoled old title with changing. So his Song Poems of Yue Fu exist of some characteristics of musical melody. Obviously, this kind of musical feeling and existing is influenced by music especially Tang Dynasty's music.

\section{InStANCE TO Old Music SCORE OF Li BAi POEM}

Although tune of Ancient musical office (Yue $\mathrm{Fu}$ ) already had many lost to the Tang Dynasty, but still left more than sixty old songs. Li Bai musical office (Yue Fu) poetry such as Long lovesickness (poem, Chang Xiang Si), Mountain Crane Pigeon Word (poem, Shan He Ge Ci), Black Night Crow (poem, Wu Ye Ti), Pick Lotus Song (poem, Cai Lian Ge) and so on all are name of Tang dynasty musical song. Old title Ancient musical office (Yue Fu) poetry such as Song of Middle-Night (poem, Zi Ye Ge), Black Night Crow (poem, Wu Ye Ti), still may be sang because of existing past tune. Again for example Difficult Shu Road (poem, Shu Dao Nan), Long lovesickness (poem, Chang Xiang Si), in poem full of flying and moving image, surging passion, fluttering and spiraling melody, and has musical innervation and power of stimulating heart.

\section{A. Finding Road of AMS (Antique Music Score) of Li Bai Poem}

After query of Li Bai poem datum, found his poems revealed large number traces of descripting music. These marks overtly or covertly, or instrument or dance or sing or crowd chorus alone. Among them, poems about old lyre (Gu qin) have 42 songs. Poems are collect and record into Poetry Anthology of Musical office (Yue Fu Shi Ji) by Guo Maoqian have163 songs. Poem of using song as title have 69 songs [6].

Besides of Ancient musical office (Yue $\mathrm{Fu}$ ) poem of $\mathrm{Li}$ Bai, large number poems are not collected and recorded into Ancient musical office (Yue Fu) Anthology such as using singing, chanting, dancing, musical instrument as title of poem. Li Bai love old lyre (Guqin) and have plentiful musical understanding and feeling to old lyre (Guqin) is inferred from appearing frequency of musical instrument in poem.

Above11songs of AMS in Li Bai poem was found by query of Chinese ancient books. These evidences that li bai poems can be sung, Li Bai poem has obvious characteristics of music elements. Founding of AMS provided new angle of view in researching on $\mathrm{Li}$ Bai poem musical characteristics and literature influence.

\section{B. Sample of AMS (Antique Music Score) of Li Bai Poem}

$\mathrm{Li}$ Bai whole life traveled all over the world, natural scenery of changing magnificent inspired his writing inspiration, wrote down thousands of popular good poetry. Whether Tang Dynasty or followed people all are proud of researching, chanting, singing his poetry works. Above mentioned 11songs Li Bai poem AMS (Antique Music Score), including 3 songs $\mathrm{Li}$ Bai poem AMS (Antique Music Score) cannot be translated into music score as variety of complex reasons, so only provided 8 songs Li Bai poem AMS (Antique Music Score) in article. 
For example, Example 1 of AMS (Antique Music Score) is Long Leaving Off (poem, Jiu Bie Li). This poem is collected and received into Complex Word (Zha Yan Qu Ci) of Poetry Anthology of Musical Office (Yue Fu Shi Ji). Origin of Example 1 is founded in come from Lyre Score DongGao (Dong Gao Qin Pu), and Lyre Score was decided by Wang Di. The tune belong to A feather ( $\mathrm{Yu}$ ) tune style, rhythm is $2 / 4$, form of Complex Word (zha yan).

The Example 2 of AMS (Antique Music Score) is Recollect Qin E (poem, Yi Qin E). This poem come from Chord Song Elegant Rhyme (Xian Ge Ya Yun, Zhong Hua Book Company, 2007 edition) is written by Wang Di. Origin of Example 2 is founded in Lyre Score Dong Gao (Dong Gao Qin Pu), and Musical score was decided by Wang Di. The tune belong to Palace (Gong) tune style, two paragraph body, is $\mathrm{A}+\mathrm{B}$ musical passage.

Example 3 of AMS (Antique Music Score) is Middle Night Wu Song-Autumn Song (Zhi Ye Wu Ge-Qiu Ge). This song belongs to tune of Qing Shang $\mathrm{Qu} \mathrm{Ci}$ in Poetry Anthology of Musical office (Yue Fu Shi Ji). Origin of Example 3 is founded in Lyre Score Dong Gao (Dong Gao Qin $\mathrm{Pu}$ ), and Musical score was decided by Wang Di. The tune belongs to a feather $(\mathrm{Yu})$ tune style, single paragraph style. It is consisted of three musical sentences, every musical sentence have 4 little bars. Li Bai poem, Long Leaving Off(poem, Jiu Bie Li), Long Leaving Off(poem, Jiu Bie Li), Middle Night Wu Song -Autumn Song (Zhi Ye Wu Ge-Qiu Ge), all selected from Lyre Score Dong Gao (Dong Gao Qin Pu). Lyre Score Dong Gao (Dong Gao Qin Pu) is a Lyre Musical collection be translated and spread by Xin Yue Chan master after he reached Japan in1676. And its lyrics (song word) most are literary works of past dynasties in China. Except in devoting Buddhism, he also introduces Chinese old lyre art to Japanese.

Middle Night Wu Song -Autumn Song: A slip of the moon hangs over the capital; Ten thousand washing-mallets are pounding; and the autumn wind is blowing my heart, for ever and ever toward the Jade Pass.... Oh, when will the Tartar troops be conquered, And my husband come back from the long campaign!

Example 4 of AMS (Antique Music Score) is Qing Ping Music (Qing Ping Yue). This tune belongs to near modern times tune (Qu Ci), and is selected from of Chinese Ancient Song be translated and edited by Sun Xuan Ling and Liu Dong Sheng [10]. Origin of Example 4 is founded in Music Score of Place for Borrowing Cloud (poem, Jie Yun Guan $\mathrm{Qu} \mathrm{Pu}$ ). This song belongs to D Palace (Gong) tune style, three paragraph body, rhythm is rubato (Shan ban). Three sections of postures, the second phrase of musical tune appear turning tune, turn to B feather $(\mathrm{Yu})$ tune style. Music Score of Place for Borrowing Cloud (Jie Yun Guan Qu Pu) is very trouble seen of Gong Che notation (Gong $\mathrm{Ci} \mathrm{Pu}$, a traditional Chinese muscial notation) of folk song album. It is edited by HuaQiuPing (Wuxi city, 1784-1859) and published in 23 year of JiaQing (in 1818).

Example 5 of AMS (Antique Music Score) is Moon of Guan Mountain (Guan Shan Yue). This tune belongs to D Palace(Gong) tune style, three paragraph body and adding end sound, rhythm is ragtime, is style of " $\mathrm{A}+\mathrm{B}+\mathrm{C}+$ end sound". Origin of Example 5 is founded in Plum Blossom Nunnery Lyre Score (Mei An Qin Pu), musical score was decided by Wang Yan Qing.

Example 6 of AMS (Antique Music Score) is Wine Beard (Jiu Hu Zi) [11]. Origin of Example 6 is founded in Important Record of Benevolence and Wisdom (Ren Zhi Yao Lu). This tune belongs to D Palace (Gong) tune style, one paragraph body, it is consisted of four musical sentences.

Example 7 of AMS (Antique Music Score) is White Zhu (poem, Bai Zhu). This tune belongs to Dance Tune Song Rhetoric of Poetry Anthology of Musical office (Yue Fu Shi Ji). Origin of Origin of Example 7 is founded in Important Record of Benevolence and Wisdom (poem, Ren Zhi Yao $\mathrm{Lu})$. This tune belongs to $\mathrm{D}$ Zhi(Zhi) tune style, two paragraph body, is musical paragraph style of $\mathrm{A}+\mathrm{A}$.

Example 8 of AMS (Antique Music Score) is Autumn Wind Rhetoric (poem, Qiu Feng Ci). Origin of Example 8 is founded in Plum Blossom Nunnery Lyre Score (poem, Mei An Qin Pu). Musical score was played and sang by Wang duan rong, and was recorded by Wang di. This song rhetoric adopted sentence style of three, five, seven word. So it have characteristic of fresh, lively and easy join music.

Wine Beard (Jiu Hu Zi) and White Zhu (poem, Bai Zhu) of $\mathrm{Li}$ Bai poem is selected from Important Record of Benevolence and Wisdom (Ren Zhi Yao Lu). Important Record of Benevolence and Wisdom (Ren Zhi Yao Lu) is one kind of Collection of Zheng Music Score, and it recorded many music works of Tang Dynasty while them spreading Japan. Zui Qi Yan Zhi, YueXia DuZhuo and Mu $\mathrm{Yu} \mathrm{Zi}$ of Li Bai poem is collected into Lyre Score Wei Shi (Wei Shi Yue Pu). Although Antique Music Score of the three poems appeared some information of culture exchange with Japanese culture, but they is not be translated into Five Staff because of complex history reasons. Original Antique Music Score the three poems are showed in here (Zui Qi Yan Zhi, YueXia DuZhuo and Mu Yu Zi.). These lyres are the composition of Gong Che Notation (Gong Ci Pu), Chinese word, Japanese katakana alphabet. According to the habits of Japanese pronunciation, may judge these lyres for Japanese application. At the same time, historical records, the end of Chongzhen (about in 1644), Lyre Score Wei Shi (Wei Shi Yue $\mathrm{Pu}$ ) is one kind of collection of popular ancient songs lyre in the palace. Musical officer of palace, Wei Shuang Hou (name, Zi Yan) take refuge in Japan, settled in Nagasaki (Japan).His IV grandson, Wei Hao (name, Zi Ming), teach the ancestral Chinese ancient songs to Japanese students. In order to facilitate of teaching, choice 200 songs edit to a book, named Lyre Score Wei Shi (Wei Shi Yue Pu). Today only existed 50 songs, the contents of The Book Songs, HanYueFu and Tang poems. From Lyre Score Wei Shi (Wei Shi Yue $\mathrm{Pu}$ ) further concluded that Li Bai poetry in ancient can be sung, and show obvious characteristics of music. 


\section{MusicAl CharaCteristics OF Li BAi AMS (ANTIQUE MUSIC SCORE)}

\section{A. Form of Singing with Song Companying Dance}

Early on, poetry, song, dance, music is whole unit. Li Bai poem form in the Tang Dynasty exist by three-dimensional pattern including poetry, singer, dancer, player of musical instrument, appreciator. A large number of them perform with orchestra accompany and dancers accompany, so while reading poetry showed some musical effect. For example, Alone Drinking under Month (poem, Yue Xia Du Zhuo), moon pace up and down while I sing, shadow is disorder while I dance. In this poem express scenes such as singing, dancing, reciting. In Ancient Wind (Gu Feng) No.21i, it clearly described singing this poetry situation. That is say that only single sing without musical instruments accompanying, singing song have folk song style of Shu region(Si Chuan province of China), Xiang (one kind style of dance) and singing style be used, one sing and thousand person act in cooperation with each other. In Walk Spring Day (Li Bai poem, Chun Ri Xing), thriving and peace scene of king palace are clearly described. In Walk from Thistle North Door (Li Bai poem, Chu Zi Ji Bei Men Xing), Trouble of Walking Road (Li Bai poem, Xing Lu Nan) No.2, Sword Song-Trouble of Walking Road (Li Bai poem, Jian Ge-Xing Lu Nan), See White Hair old Man in Weeds (Li Bai poem, Jian Ye Cao Zhong You Yue Bai Tou Weng), These poems reflected Li Bai love sing all cannot control his feelings on way back home, in playing sword, in wilderness, in forest, after drunk, and love sing don't distinguish situation. These describing sentence fully indicated Li Bai love sing.

\section{B. Musical Rhythm with Surroundings Changing}

Some long song poem musical office (Yue Fu ) of Li Bai, $\mathrm{Da}$ qu and $\mathrm{Fa}$ qu of Flourishing Tang dynasty China, their layout structure and rhythm have abundant changing, such characteristic can be found in Difficult Shu Road (poem, Shu Dao Nan). See from layout structure, Difficult Shu Road like a song magnificent symphonic music, structure is very intact and full changing. Main tune and changing rhythm, plentiful changing and unimpeded topic have been embodied in poem. Trouble of Shu road is more difficult than Climb to Sky, this sentence appeared beginning, middle and end, of poem, such emphasized theme of poem and role of one singing three plaint. In rhythm and rhyme, neither orderliness and symmetrical sentence or full long and short changing of scattered sentence in Difficult Shu Road (poem, Shu Dao Nan). And steady rhythm of Five word and Seven word, rapid rhythm of Three word and Four word, smooth and relaxed of Nine word and Eleven word, these characteristic described dynamic beauty of music flow changing.

\section{Dance Styles of Ethnic Characteristic}

Music and dance of Flourishing Tang dynasty China absorb a large number of national minority, foreign music and dance elements, especially music and dance of Central Asia and Western regions, rhythm and the melody all are very powerful and rapid. For example Hu Revolving dance and $\mathrm{Hu}$ Jumping dance all have characteristic as rapid rotating like flying, exciting and be lively. Fresh blood and artistic vigor was input into Han national music and dance with slow rhythm and mild melody. So song and dance is more colorful in Flourishing Tang dynasty than any one other dynasty before. From Sky Horse Song (poem, Tian Ma $\mathrm{Ge}$ ), we can find out a kind of related to these characteristics of basic syllables.

Even has researcher think that poetry of Qinghai wave dance music is one of $\mathrm{Li}$ Bai famous songs. It is interesting that tones in Qinghai wave dance music again be copied out from Japanese Tang music library by Pi ken is astonishing similarities with Central Asian music we already know it today. This explain that Li Bai ability in be good at describe and abundant summoning very suit for be sang poetry, and particularly adapt for eighth century music. Some Li Bai poem rhyme and rhythm is obviously connected with music and dance of Central Asia or western region. Music and dance of Central Asia or western region is warm and lively, changeable rhythm. These factors effect on Li Bai poem. Especially its influence is especially striking in Li Bai's Complex Word- song poem musical office (Yue Fu) (Za Yan, a type of classical Chinese poetry) and Word ( $\mathrm{Ci}$, a type of classical Chinese poetry) created long and short sentence of Music Office poetry.

\section{Musical Value in singing AMS (Antique Music Score) of Li Bai Poem}

Prosperity of poetry and music propelled the development of political and economic in Tang Dynasty China. Qu XiaoHong (2014) pointed out that very high level of writing song techniques and Chinese musical elements all were used such as Chinese opera, Chinese traditional musical instruments, Chinese pentatonic scales (five-tone style) in the article, Analysis of the work style and singing of Lin Lepei's vocal suite "Three Poems about Night by Li Bai" by Mr Lin Le Pei [9-11]. The relationship between poetry and music people of Tang Dynasty is researched in the article, analysis on works style and singing in vocal music suite song of three song Li Bai nigh poetry [12].

Consult related books of $\mathrm{Li}$ Bai poem, singing and playing techniques in Tang Dynasty poetry are summed up and summarized. And be compared singing music skills in contemporary times and Tang Dynasty. Some advantages and national singing style are referenced basing on summarized researching achievement in Li Bai poem. The spread and development of Li Bai poem and music enlighten us bring into play the modern value of music culture to propel political, economic and cultural construction and to promote connotation of Chinese nation culture.

\section{CONCLUSION}

$\mathrm{Li}$ Bai poem can be sang, it reflects the music connotation of poetry and musical trace records inheriting process of Chinese culture of Tang Dynasty. Be found a few AMS (Antique Music Score) of Li Bai poem proved the intimate relationship between poetry and song. Because of original relationship of $\mathrm{Li}$ Bai poem and Antique Music Score, Li Bai poem can be widely spread and keeping fresh vitality. And repeated singing AMS (Antique Music Score) can reappear history and cultural ecology of the Tang 
Dynasty be accompanied to provide original elements and new path on research Li Bai.

\section{REFERENCES}

[1] Xu YuanChong. Selected poetry of Li Bai (English translation album) $[\mathrm{M}]$. Beijing, China Translation \&Publishing Corporation, 2014-5.

[2] Cayne, Bernard S., et al. The Encyclopedia Americana [M]. Grolier, Inc., 1981.

[3] Gautier J. Le Livre de jade[M]. A. Lemerre, 1867.

[4] Ge Jing Chun. Li Bai poem and tang music[J].Literary heritage(in Chinese) ,1995(03): 43-51.

[5] Hou Yanshuang. Music and singing of Li Bai poem[J]. Journal of CHINESE MUSIC(in Chinese), 2009(03): 175-185.

[6] Hou Yanshuang. Music of Li Bai poem [J]. Journal of Music creation(in Chinese) ,2009(04): 106-109.

[7] Zhao Lu. A Comparative Study of Music in Li Bai's and Wang Wei's Yuefu Poetry. Journal of Mianyang Normal University(in Chinese), 2013(09): 04-08.

[8] Fu Xiao lin, Zhao Lu. The Music in Li Po's "Musical Poem" of Yuefu [J].Journal of Mianyang Normal University(in Chinese), 2013(07) : 31-33

[9] Qu XiaoHong. Analysis of the work style and singing of Lin Lepei's vocal suite <Three Poems about Night by Li Bai>[D]. Shang hai, SHANGHAI CONSERVA-TORY OF MUSIC, 2014.

[10] Sun Xuan Ling, Liu Dong Sheng. Chinese Ancient Song [M]. Beijing, People's Music Publishing House, 1990.

[11] Guan Ye We. Music History of Tang Dynasty [M]. Beijing, the press of Minzu University of China, 2006.

[12] Hai Jun.The study of LiBai's Yuefu poem[D]. Lan zhou, Northwest Normal University, 2010. 